\title{
Análise da produção científica sobre estilos de aprendizagem e uso de tecnologias
}

\author{
Rodrigo Hipólito Roza ${ }^{1}$; Solange Muglia Wechsler ${ }^{2}$
}

\begin{abstract}
Resumo: Este estudo apresenta uma análise exploratória da produção científica sobre estilos de aprendizagem em situações de uso das Tecnologias da Informação e Comunicação (TICs). Foram analisados artigos de periódicos revisados por pares, no período de 2010 a 2015, obtidos a partir do Portal de Periódicos da Coordenação de Aperfeiçoamento de Pessoal de Nível Superior (CAPES). As buscas foram realizadas com os termos "learning styles" e "technology", e seus equivalentes em português e espanhol. Em inglês, foram encontradas 209 publicações sobre estilos de aprendizagem, sendo que $28(13,40 \%)$ delas estavam relacionadas ao uso de tecnologias. Em português, foram apenas duas publicações sobre estilos de aprendizagem, mas nenhuma delas estava relacionada ao uso de tecnologias. Já em espanhol, foram 17 publicações sobre estilos de aprendizagem, sendo duas $(11,76 \%)$ relacionadas ao uso de recursos tecnológicos. Houve uma diversidade alta de assuntos relacionados ao uso de tecnologias, com predominância de publicações sobre e-learning. Tais constatações, incluindo a baixa quantidade relativa de publicações sobre estilos de aprendizagem em situações de uso de tecnologias, sugerem a necessidade de mais estudos sobre o tema.
\end{abstract}

Palavras-chave: estilos de aprendizagem, tecnologia, tecnologia da informação e comunicação, produção científica.

\section{Analysis of scientific production on learning styles and technology use}

\begin{abstract}
This study presents an exploratory analysis of scientific literature on learning styles in situations of Information and Communication Technologies (ICTs) use. Journal articles peer reviewed were analyzed, from 2010 to 2015, obtained from the Portal of Journals of Higher Education Personnel Improvement Coordination (CAPES). The searches were conducted with the terms "learning styles" and "technology" and its equivalent in Portuguese and Spanish. In English, were found 209 publications on learning styles, and 28 (13.40\%) of them were related to technology use. In Portuguese, there were only two publications on learning styles, but none of them was related to technology use. Already in Spanish were 17 publications on learning styles, two $(11.76 \%)$ related to the use of technological resources. There was a high diversity of issues related to technology use, with a predominance of publications on e-learning. These findings, including the relatively low number of publications on learning styles in technology use situations, suggest the need for further studies on the subject.
\end{abstract}

Keywords: learning styles, technology, information and communication technology, scientific production.

\footnotetext{
${ }^{1}$ Doutor em Psicologia e Mestre em Ciência da Informação pela Pontifícia Universidade Católica de Campinas. Bacharel em Ciência da Computação pela Universidade Paulista. Professor do Centro de Economia e Administração da Pontifícia Universidade Católica de Campinas, São Paulo, Brasil, e-mail: rodrigo.roza@gmail.com.

${ }^{2}$ Doutora e Mestre em Psicologia pela University of Georgia. Psicóloga pela Pontifícia Universidade Católica do Rio de Janeiro. Pósdoutorado pela University of Georgia e pela University of Buffalo. Professora do Programa de Pós-Graduação em Psicologia da Pontifícia Universidade Católica de Campinas, São Paulo, Brasil, e-mail: wechsler@lexxa.com.br.
} 


\section{Introdução}

A produção científica sobre estilos de aprendizagem é vasta e vem sendo desenvolvida e aplicada nas mais variadas situações. Dentre as diversas pesquisas neste campo, é possível destacar os trabalhos de autores como Dunn e Dunn (1978), Kolb (1984), Felder e Silverman (1988), Mumford e Honey (1992), Alonso, Gallego e Honey (1997), que ao longo das últimas décadas buscaram compreender as preferências dos indivíduos ao aprender e influenciaram as investigações científicas de vários outros pesquisadores.

Em linhas gerais, o conceito de estilo remete à ideia de preferências individuais no uso de habilidades, não podendo ser rotulados como boas ou ruins, mas apenas como diferentes (STERNBERG, 1994). Trata-se dos modos preferenciais de um indivíduo pensar e agir frente a determinadas situações (WECHSLER, 2006). Os estilos de aprendizagem, em particular, referem-se aos modos característicos empregados por um indivíduo para adquirir, conservar e recuperar informações, segundo a visão de Felder e Henriques (1995). Na concepção de Kolb e Kolb (2005, 2006), tais estilos estão relacionados às diferenças individuais de aprendizagem, baseadas nas preferências do indivíduo por diferentes fases do seu ciclo de aprendizagem.

Para conceituar estilos de aprendizagem, Santos e Wechsler (2008) destacam o modo particular empregado por um indivíduo para adquirir experiências e conhecimentos. Já Gallego (2013) enfatiza o conceito de estilo de aprendizagem como uma soma de estilo cognitivo, que dificilmente se modifica por estar mais próximo da fisiologia, com estratégias de aprendizagem, que permitem ao indivíduo variar o modo como aprende.

Várias teorias e modelos foram desenvolvidos para explicar os estilos de aprendizagem. Dunn e Dunn (1978), por exemplo, consideram os elementos ambientais, emocionais, sociológicos, fisiológicos e psicológicos em seu modelo de estilos de aprendizagem. Dessa forma, consideram: iluminação, temperatura, ruído e design de ambiente, nos elementos ambientais; responsabilidade, motivação, persistência e necessidade de estrutura, nos elementos emocionais; o aprender sozinho, em dupla, com colegas, com adultos e de forma variada, nos elementos sociológicos; horário, modalidades preferenciais de atenção, alimentação e mobilidade durante o aprender, nos elementos fisiológicos; e a distinção entre global versus analítico, reflexivo versus impulsivo, nos elementos 
psicológicos. Os autores criaram o Learning Style Inventory and Productivity Environmental Preference Survey Learning como instrumento de avaliação.

Kolb (1984), por sua vez, desenvolveu a Experiential Learning Theory - ELT, cujo modelo apresenta o processo de aprendizagem de forma cíclica, envolvendo a experiência concreta, a observação reflexiva, a conceituação abstrata e a experimentação ativa. Assim, originalmente foram identificados quatro estilos de aprendizagem divergente, assimilador, convergente e acomodador.

Conforme apontado por Kolb e Kolb (2005), no estilo divergente existe predominância da experiência concreta e da observação reflexiva. No estilo assimilador predominam a conceituação abstrata e a observação reflexiva. A conceituação abstrata e a experimentação ativa são marcantes no estilo convergente e, no estilo acomodador, a experiência concreta e experimentação ativa são as habilidades de aprendizagem dominantes. Como instrumento de avaliação, foi desenvolvido o Inventário de Estilos de Aprendizagem (Learning Style Inventory - LSI).

Já o modelo apresentado por Felder e Silverman (1988) considerou inicialmente cinco dimensões de estilos de aprendizagem. A partir desse modelo, Soloman e Felder (2001) desenvolveram o Index of Learning Styles (ILS) para avaliação dos estilos de aprendizagem. Posteriormente, o modelo foi revisto por Felder (2002), passando a conter quatro dimensões: percepção (sensorial/intuitivo), entrada (visual/verbal), processamento (ativo/reflexivo) e compreensão (sequencial/global).

Segundo Honey (1994), uma versão simplificada do modelo de Kolb (1984) foi desenvolvida por Mumford e Honey, compreendendo os estilos de aprendizagem ativo, reflexivo, teórico e pragmático. Com um enfoque empresarial, os autores criaram Learning Styles Questionnaire (LSQ). Mais tarde, tais estudos foram recorridos por Alonso, Gallego e Honey (1997), porém abordando os estilos de aprendizagem com um enfoque no mundo acadêmico. Como instrumento, os autores criaram o Cuestionario Honey-Alonso de Estilos de Aprendizaje - CHAEA (ALONSO; GALLEGO, 1995).

As teorias e os modelos de estilos de aprendizagem têm sido estudados e empregados nos mais diferentes contextos, inclusive em situações de uso das denominadas tecnologias da informação e comunicação - TICs (ROZA, 2017). Tais tecnologias podem ser compreendidas 
como o conjunto de recursos tecnológicos empregados no tratamento, na organização e na disseminação da informação (TAKAHASHI, 2000).

As tecnologias da informação e comunicação envolvem, portanto, desde computadores, tablets e smartphones, seus diversos aplicativos, ou programas, e as redes computacionais locais, até os sistemas de telecomunicações, as redes de longa distância e a própria Internet. Elas estão na base das transformações observadas na atual sociedade (COLL; MONEREO, 2010; CASTELLS, 2005, 2010), rotulada por alguns estudiosos como sociedade da aprendizagem (POZO, 2004; COUTINHO; LISBÔA, 2011).

Na educação, as tecnologias da informação e comunicação são de grande importância na criação de espaços e comunidades virtuais de aprendizagem, de forma complementar à aprendizagem presencial (MOREIRA; MONTEIRO, 2010). Porém, também podem causar efeitos indesejáveis, como barreiras à criatividade, caso os alunos não estejam adaptados às ferramentas tecnológicas (SATHLER; FLEITH, 2010).

Conforme citado anteriormente, os estudos sobre estilos de aprendizagem têm sido conduzidos em diferentes contextos. Em situações de uso dos recursos tecnológicos, são encontrados, por exemplo, estudos de autores como o de Smith (2010) que utilizou o inventário de estilos de aprendizagem de Kolb (KOLB; KOLB, 2005) para avaliar enfermeiros matriculados em um curso on-line de uma universidade dos Estados Unidos. Também existem estudos como os conduzidos por Hwang et al. (2013). Os autores instigaram a habilidade de estudantes de uma escola primária de Taiwan em escolher o sistema de $e$ learning ou apresentação de conteúdo de acordo com seus estilos de aprendizagem, usando duas versões de um jogo educacional baseado na dimensão sequencial/global do modelo de Felder e Silverman (1988).

De fato, os estudos sobre estilos de aprendizagem têm sido realizados e aplicados em variadas situações de uso das tecnologias informação e comunicação, abrangendo tópicos diversos e muitas vezes correlatos, como o próprio e-learning (DEBORAH; BASKARAN; KANNAN, 2014), ambientes virtuais de aprendizagem (KURILOVAS; KUBILINSKIENE; DAGIENE, 2014; DASCALU et.al., 2015) e aprendizagem com o uso de dispositivos móveis (SHUIB et al., 2015), por exemplo. Considerando, portanto, o impacto das tecnologias da informação e comunicação na atual sociedade e os estilos de aprender frente a esta nova 
realidade, o presente estudo busca analisar, de forma exploratória, a produção científica recente sobre as preferências individuais de aprendizagem em situações de uso de tecnologias.

\section{Método}

\section{Material}

A presente pesquisa foi realizada através dos dados sobre publicações científicas disponibilizados no Portal de Periódicos da Coordenação de Aperfeiçoamento de Pessoal de Nível Superior - CAPES (http://www.periodicos.capes.gov.br/), do Ministério de Educação

(MEC) do Brasil. É importante salientar que o portal de periódicos da CAPES foi escolhido por permitir a realização de buscas em diversas bases de dados e periódicos científicos, nacionais e internacionais, cobrindo todas as áreas do conhecimento. Tal característica foi de fundamental importância para os objetivos da pesquisa, dado o caráter multi e interdisciplinar do presente estudo, assumido ao abordar os estilos de aprendizagem especificamente em situações de uso de tecnologias.

\section{Procedimento}

No portal de periódicos da CAPES, primeiramente foram realizadas buscas por publicações com o assunto "learning styles", no período de 2010 a 2015, em periódicos revisados por pares. Posteriormente, foram buscadas publicações com o assunto "learning styles" que continham qualquer ocorrência do termo "technology", no período de 2010 a 2015, em periódicos revisados por pares. O mesmo procedimento foi repetido para os termos equivalentes em português, "estilos de aprendizagem" e "tecnologia", e em espanhol, "estilos de aprendizaje" e "tecnología".

O termo "technology" e seus equivalentes em português e espanhol foram utilizados por serem mais amplos que outros termos similares, de forma a recuperar uma quantidade maior de publicações. A partir da leitura dos resumos, foram eliminados os trabalhos que não correspondiam ao objeto deste estudo. Por fim, também por meio dos resumos, os seguintes 
aspectos foram analisados: idioma de publicação dos artigos, ano de publicação, participantes da pesquisa, assuntos relacionados à tecnologia e referenciais teóricos sobre estilos de aprendizagem.

\section{Resultados}

Nas buscas realizadas no portal de periódicos da CAPES, segundo os critérios citados anteriormente no procedimento de pesquisa, foram encontradas 228 publicações sobre estilos de aprendizagem. Dentre elas, 30 eram relacionadas a situações de uso das tecnologias da informação e comunicação, correspondendo a 13,16\% do total de publicações.

Nas buscas com o assunto "learning styles", em inglês, foram encontradas 209 publicações sobre estilos de aprendizagem, sendo 28 delas $(13,40 \%)$ em situações de uso de tecnologias. Em português, foram duas publicações sobre estilos de aprendizagem, mas nenhuma em situações de uso de tecnologias. No idioma espanhol, foram 17 publicações sobre estilos de aprendizagem, sendo duas $(11,76 \%)$ relacionadas a situações de uso de recursos tecnológicos.

Considerando apenas o subconjunto de publicações sobre estilos de aprendizagem em situações de uso de tecnologias, observa-se que 93,33\% estavam no idioma inglês. Em espanhol, este percentual foi de $6,67 \%$.

Nas publicações sobre estilos de aprendizagem em situações de uso de recursos tecnológicos, foram encontradas cinco publicações em 2010, quatro em 2011, quatro em 2012, nove em 2013, três em 2014 e cinco em 2015. As categorias dos participantes da pesquisa identificados nas publicações são apresentadas na Tabela 1.

\section{Tabela 1}

Participantes da pesquisa

\begin{tabular}{lcc}
\hline Participante & $\mathrm{n}$ & $\%$ \\
\hline Estudantes & 19 & 67,86 \\
Aprendizes & 7 & 25,00 \\
Instrutores & 1 & 3,57 \\
Profissionais & 1 & 3,57 \\
\hline
\end{tabular}


De acordo com a Tabela 1, a maioria das publicações teve estudantes e aprendizes como participantes da pesquisa. Os estudantes, neste caso, correspondem aos indivíduos em situações formais de aprendizagem; os aprendizes, por sua vez, referem-se aos indivíduos em situações informais de aprendizagem. Dentre os estudantes, nove $(47,37 \%)$ eram citados como estudantes de nível superior. Já os assuntos relacionados às tecnologias da informação e comunicação são mostrados na Tabela 2, de acordo como aparecem os termos resultantes da busca.

Tabela 2

Assuntos relacionados à tecnologia

\begin{tabular}{lcr}
\hline Assunto & $\mathrm{n}$ & \multicolumn{1}{c}{$\%$} \\
\hline E-Learning & 5 & 13,89 \\
Distance Learning & 2 & 5,56 \\
Educational Computer Games & 2 & 5,56 \\
Internet & 2 & 5,56 \\
Technology & 2 & 5,56 \\
Virtual learning environments & 2 & 5,56 \\
Adaptive e-learning systems & 1 & 2,78 \\
Adaptive hypermedia & 1 & 2,78 \\
Competencias Digitales & 1 & 2,78 \\
Computer Programming & 1 & 2,78 \\
Digital Natives & 1 & 2,78 \\
Educational Recommender System & 1 & 2,78 \\
Educational systems & 1 & 2,78 \\
E-learning contents & 1 & 2,78 \\
E-learning server & 1 & 2,78 \\
ICT's for human capital & 1 & 2,78 \\
Information Technology & 1 & 2,78 \\
Mobile Learning & 1 & 2,78 \\
Multimedia learning & 1 & 2,78 \\
Online Faculty & 1 & 2,78 \\
Online learning & 1 & 2,78 \\
Online Learning Environments & 1 & 2,78 \\
Online Systems Design & 1 & 2,78 \\
Recursos Educativos Digitales & 1 & 2,78 \\
Technology-Enhanced Collaborative Learning & 1 & 2,78 \\
Virtual environments & 1 & 2,78 \\
Web-based education & 1 & 2,78 \\
\hline
\end{tabular}


É importante ressaltar que, na Tabela 2, os termos foram intencionalmente mantidos no idioma original das publicações, para uma maior precisão na apresentação dos resultados. $\mathrm{Na}$ sequencia, contudo, os termos foram traduzidos, tendo em vista sua posterior discussão. Quando pertinente, os termos foram mantidos sem tradução por serem considerados amplamente difundidos, como no caso do termo e-learning, ou ainda foram comentados para situá-los no idioma português.

De acordo com a Tabela 2, foram identificadas mais de uma ocorrência dos seguintes assuntos relacionados aos recursos tecnológicos: e-learning, ensino à distância, jogos educativos de computador, Internet, tecnologia, ambientes virtuais de aprendizagem. Com apenas uma ocorrência cada, também foram identificados: sistemas adaptativos de e-learning, hipermídia adaptativa, competências digitais, programação de computadores, nativos digitais, sistema de recomendação educacional, sistemas educacionais, conteúdos de e-learning, servidor de e-learning, TICs para o capital humano, tecnologia da informação, aprendizagem móvel, aprendizagem multimídia, faculdade online, aprendizagem online, ambientes de aprendizagem online, design de sistemas online, recursos educativos digitais, tecnologia avançada de aprendizagem colaborativa, ambientes virtuais e educação baseada na Web.

Cabe destacar que os ambientes virtuais de aprendizagem citados em algumas publicações são frequentemente referenciados pela sigla "AVA", na literatura em língua portuguesa. A abreviação ICT's encontrada em um dos artigos significa Information and Communication Technologies ou, em português, Tecnologias da Informação e Comunicação (TICs).

Os dados sobre os referenciais teóricos sobre estilos de aprendizagem, adotados nas publicações também foram verificados. Os resultados encontram-se na Tabela 3. 
Tabela 3

Referenciais teóricos sobre estilos de aprendizagem

\begin{tabular}{lcr}
\hline Referencial teórico & $\mathrm{n}$ & $\%$ \\
\hline Modelo de estilos de aprendizagem de Felder e Silverman & 5 & 16,67 \\
Myers Briggs Type Indicator - MBTI & 3 & 10,00 \\
Inventário de estilo de aprendizagem de Kolb & 2 & 6,67 \\
Teorias/modelos de estilos de aprendizagem & 3 & 10,00 \\
Não disponível & 17 & 56,67 \\
\hline
\end{tabular}

De acordo com a Tabela 3, no que se refere aos referenciais teóricos sobre estilos de aprendizagem, foram encontradas menções ao modelo de Felder e Silverman, ao Myers Briggs Type Indicator (MBTI) e ao inventário de estilo de aprendizagem de Kolb. Nas publicações, também foram encontradas menções a "teorias/modelos de estilos de aprendizagem" de uma forma genérica, sem, no entanto, explicitá-las no resumo.

\section{Discussão}

A quantidade de publicações sobre estilos de aprendizagem em situações de uso de tecnologias mostrou-se baixa frente à quantidade total de publicações sobre estilos de aprendizagem. Em situações de uso de tecnologias, os artigos publicados em inglês foram maioria, seguida dos artigos em espanhol. Já em língua portuguesa, não foram identificados artigos segundo os critérios pré-estabelecidos no método deste estudo. Apesar da grande diferença do número de publicações em inglês e espanhol, observa-se que as razões entre a quantidade de publicações sobre estilos de aprendizagem em situações de uso de tecnologias e a quantidade total de publicações sobre estilos de aprendizagem foram similares nos dois idiomas.

A distribuição das publicações sobre o tema foi mais constante de 2010 a 2012 e no ano 2015, apresentado um pico em 2013 e um decréscimo em 2014. Contudo, por se tratar de 
uma quantidade baixa de publicações, não é possível afirmar que houve maior ou menor interesse sobre a temática ao longo desse período.

A maior parte das publicações teve estudantes como participantes da pesquisa, sendo que quase metade delas estava voltada a estudantes de nível superior. Tal constatação indica uma preocupação com o estudo dos estilos de aprendizagem e uso dos recursos tecnológicos em ambientes formais de aprendizagem, notadamente no âmbito acadêmico. $\mathrm{O}$ fato de quase metade das publicações ter estudantes de nível superior como participantes, por outro lado, pode ser reflexo de uma maior proximidade dos pesquisadores com os participantes das pesquisas, pois muitos estudos são desenvolvidos por pesquisadores ligados a universidades. Porém, também é importante destacar a ocorrência de estudos com indivíduos em ambientes informais de aprendizagem, uma vez que a aprendizagem não se restringe às escolas ou às universidades.

A variedade de assuntos relacionados à tecnologia, vista como os recursos tecnológicos usados para tratar, organizar e disseminar a informação (TAKAHASHI, 2000), mostrou-se alta, remetendo tanto a questões tecnológicas em si, como a questões mais sistêmicas sobre estilos de aprendizagem e o uso de recursos tecnológicos. Os assuntos relacionados à tecnologia mostram que houve predominância de publicações sobre $e$-learning . Neste sentido, também foram encontradas ocorrências como sistemas adaptativos de $e$ learning, conteúdos de e-learning e servidor de $e$-learning.

É importante destacar que os assuntos relacionados às tecnologias da informação e comunicação foram apresentados de forma não categorizada no presente estudo, pois alguns deles poderiam ser considerados em mais de uma categoria. É o caso, por exemplo, dos sistemas adaptativos de e-learning, que poderiam pertencer tanto à categoria de e-learning como à categoria de sistemas adaptativos.

A maioria das publicações encontradas não cita, em seu resumo, o referencial teórico sobre estilos de aprendizagem utilizado. As poucas citações foram para o modelo de estilos de aprendizagem de Felder e Silverman, desenvolvido a partir das pesquisas dos autores voltadas a estudantes de engenharia (FELDER; SILVERMAN, 1988), ao Myers Briggs Type Indicator (MBTI) e ao inventário de estilo de aprendizagem de Kolb, baseado na Experiential Learning Theory (KOLB, 1984). Apesar do MBTI aparecer nas publicações pesquisadas, a própria 
Fundação Myers \& Briggs o denomina inventário de personalidade, cujo objetivo é deixar a teoria dos tipos psicológicos de Jung (JUNG, 2012/1921) mais acessível.

\section{Considerações finais}

O presente estudo foi exploratório e teve um caráter multi e interdisciplinar. Assim, optou pelo uso de um portal de periódicos com maior alcance para realizar buscas em várias bases de dados e periódicos científicos, tanto nacionais como internacionais. Também recorreu ao uso de termos abrangentes, em inglês, português e espanhol, para realização das buscas, como forma de obter resultados mais representativos para a pesquisa. Uma limitação deste estudo é o fato de ter considerado apenas artigos de periódicos revisados por pares. Uma busca mais ampla que contemplasse outros artigos ou ainda dissertações e teses, embora menos rigorosa, poderia revelar outros interesses sobre o tema.

De um modo geral, a partir das publicações analisadas, em especial no que se refere à variedade de assuntos sobre tecnologias, foi possível constatar a relevância e a vasta aplicação dos estilos de aprendizagem em situações de uso de tecnologias. Por fim, cabe destacar que a baixa quantidade relativa de publicações sobre estilos de aprendizagem em situações de uso das tecnologias da informação e comunicação, bem como a alta variedade de assuntos sobre tecnologias, identificados nas publicações analisadas, sugerem a necessidade de mais estudos sobre a temática.

\section{Referências}

ALONSO, C. M.; GALLEGO, D. J. Cuestionario Honey-Alonso de estilos de aprendizaje CHAEA, $1995 . \quad$ Disponível em: http://einnova.inf.utfsm.cl/docs/anexo1_23_sept_2004_CHAEA1.pdf. Acessado em: 27 mar. 2016.

ALONSO, C. M.; GALLEGO, D. J.; HONEY, P. Los estilos de aprendizaje: procedimientos de diagnostico y mejora. Bilbao: Ediciones Mensajero, 1997. 
CASTELlS, M. A Sociedade em Rede: do Conhecimento à Política. In: CASTELLS, M.; CARDOSO, G. (Orgs.). A Sociedade em Rede: Do Conhecimento à Acção Política. Lisboa: Imprensa Nacional - Casa da Moeda, 2005. p. 17-30.

CASTELLS, M. The rise of the network society: The information age: Economy, society, and culture. Oxford: Wiley-Blackwell., 2010.

COLL, C.; MONEREO, C. Educação e aprendizagem no século XXI: novas ferramentas, novos cenários, novas finalidades. In: COLL, C.; MONEREO, C. (Orgs.). Psicologia da educação virtual: aprender e ensinar com as tecnologias da Informação e da Comunicação. Porto Alegre: Artmed, 2010. p. 15-46.

COUTINHO, C. P.; LISBÔA, E. S. Sociedade da informação, do conhecimento e da aprendizagem: desafios para educação no século XXI. Revista de Educação, v. 18, n. 1, p.5$22,2011$.

DASCALU, M. et al. A recommender agent based on learning styles for better virtual collaborative learning experiences. Computers in Human Behavior, v. 45, p. 243-253, 2015.

DEBORAH, L. J.; BASKARAN, R.; KANNAN, A. Learning styles assessment and theoretical origin in an E-learning scenario: a survey. Artificial Intelligence Review, v. 42, n. 4, p. 801-819, 2014.

DUNN, R. S.; DUNN, K. J. Teaching students through their individual learning styles: A practical approach. Reston: Prentice Hall, 1978.

FELDER, R. M. Author's preface to learning and teaching styles in engineering education [Electronic Version]. Engr. Education, 78 (7), 674-681 (1988), 2002.

FELDER, R. M.; HENRIQUES, E. R. Learning and teaching styles in foreign and second language education. Foreign Language Annals, v. 28, n. 1, p. 21-31, 1995.

FELDER, R. M.; SILVERMAN, L. K. Learning and teaching styles in engineering education. Engineering Education, v. 78, n. 7, p. 674-681, 1988.

GALLEGO, D. J. Ya he diagnosticado el estilo de aprendizaje de mis alumnos y ahora ¿qué hago?. Journal of Learning Styles, v. 12, n. 12, p. 1-15, 2013.

HONEY, P. Styles of learning. Handbook of Management Development. Aldershot: Gower, 1994.

HWANG, G. et al. A learning style perspective to investigate the necessity of developing adaptive learning systems. Journal of Educational Technology \& Society, v. 16, n. 2, p. 188-197, 2013. 
JUNG, C. G. Tipos psicológicos. Editora Vozes, 2012 (Trabalho original publicado em 1921).

KOLB, A. Y.; KOLB, D. A. The Kolb Learning Style Inventory - Version 3.1: 2005 Technical Specifications. Boston: Hay Resource Direct, 2005.

KOLB, A. Y.; KOLB, D. A. Learning styles and learning spaces: A review of the multidisciplinary application of experiential learning. Theory in Higher Education, p. 45-91, 2006.

KOLB, D. A. Experiential learning: Experience as the source of learning and development. New Jersey: Prentice-Hall, 1984.

KURILOVAS, E.; KUBILINSKIENE, S.; DAGIENE, V. Web 3.0-Based personalisation of learning objects in virtual learning environments. Computers in Human Behavior, v. 30, p. 654-662, 2014.

MOREIRA, J. A.; MONTEIRO, A. O trabalho pedagógico em cenários presenciais e virtuais no ensino superior. Educação, Formação e Tecnologias, v. 3, p. 82-94, 2010.

MUMFORD, A.; HONEY, P. Questions and answers on learning styles questionnaire. Industrial and Commercial Training, v. 24, n. 7, 1992.

POZO, J. I. A sociedade da aprendizagem e o desafio de converter informação em conhecimento. Pátio: Revista Pedagógica, n. 31, p. 8-11, 2004.

ROZA, R. H. Estilos de aprendizagem e o uso das tecnologias da informação e comunicação. 2017. 157p. Tese (Doutorado em Psicologia como Profissão e Ciência) Pontifícia Universidade Católica de Campinas, Centro de Ciências da Vida, Programa de PósGraduação em Psicologia, Campinas, 2017.

SANTOS, E.; WECHSLER, S. Compreensão e consideração dos professores sobre estilos de aprender. Boletim - Academia Paulista de Psicologia, v. 28, n. 1, p. 72-78, 2008

SATHLER, T. C.; FLEITH, D. D. S. Estímulos e barreiras à criatividade na educação a distância. Estudos de Psicologia (Campinas), v. 27, n. 4, p. 457-466, 2010.

SHUIB, M. et al. Designing an Intelligent Mobile Learning Tool for Grammar Learning (iMoL). iJIM, v. 9, n. 1, p. 41-46, 2015.

SMITH, A. Learning styles of registered nurses enrolled in an online nursing program. Journal of Professional Nursing, v. 26, n. 1, p. 49-53, 2010.

SOLOMAN, B. A.; FELDER, R. M. Index of learning styles questionnaire, 2001. Disponível em http://www.engr.ncsu.edu/learningstyles/ilsweb.html. Acesso em: 16 out. 2015 
Id on Line Revista Multidisciplinar e de Psicoloqia

Id on Line Multidisciplinary and Psycology Journal

STERNBERG, R. J. Allowing for Thinking Styles. Educational Leadership, v. 52, n. 3, p. 36-40, 1994.

TAKAHASHI, T. (Org.). Sociedade da informação no Brasil: livro verde. Ministério da Ciência e Tecnologia, 2000.

WESCHSLER, S. M. Estilos de Pensar e Criar: impacto nas áreas educacional e profissional. Psicología, cultura y sociedad, v. 7, p. 207-218, 2006.

\section{Como citar este artigo (Formato ABNT):}

ROZA, Rodrigo H.; WECHSLER, Solange M. Análise da produção científica sobre estilos de aprendizagem e uso de tecnologias. Id on Line Revista Multidisciplinar e de Psicologia, 2017, vol.11, n.37, p.13-26. ISSN: 1981-1179.

Recebido: 02.08.2017

Aceito: 07.08. 2017 